\title{
Effect of feedback on test ordering behaviour of general practitioners
}

\author{
R A G Winkens, P Pop, R P T M Grol, A D M Kester, J A Knottnerus
}

\begin{abstract}
Objective-To assess the effects of feedback on the test ordering behaviour of general practitioners.

Design-Comparison of requests at two diagnostic centres, and internal comparison between tests which were discussed in feedback and tests which were not.
\end{abstract}

Setting-A diagnostic centre in Maastricht giving feedback and another elsewhere in the Netherlands (laboratory A) not giving feedback.

Subjects-All 85 general practitioners in the region of Maastricht, and all general practitioners in the region of laboratory $A$.

Main outcome measures-Numbers of tests requested by general practitioners.

Results-Requests at the Maastricht diagnostic centre decreased soon after the onset of feedback whereas there was a persistent increase in requests at laboratory A. Tests that were discussed showed the strongest decrease (maximum 40\%), though tests that were not discussed decreased as well (maximum 27\%).

Conclusions-Feedback on diagnostic requests may exert a strong influence on request behaviour. Four years after the onset of feedback the effects were still noticeable.

\section{Introduction}

For many years there have been efforts in primary and secondary health care to influence the behaviour of physicians. ${ }^{1.5}$ One method is to provide feedback,${ }^{6}$ and the effects have been extensively studied. ${ }^{7-16}$ Results are generally positive, although the effects are usually temporary. ${ }^{81113-16}$ In reported series, however, feedback was offered mainly within the setting of scientific studies with limited duration.

The diagnostic centre in Maastricht provides feedback as a health care activity. ${ }^{17}$ Uniquely based on the request forms filled in by the general practitioner, comments are made about inappropriate requests or recommendations are offered with regard to rational diagnosis. Thus the feedback concerns real cases from daily general practice.

Overviews and estimations of the Maastricht diagnostic centre data indicated that the feedback was accompanied by a reduction in the total number of tests ordered in subsequent years. In these first analyses no account was taken of factors such as the intensity of the information provided in the feedback (the more frequently a subject is discussed the greater is the effect expected) and the request trends which would have occurred in the absence of feedback. These considerations prompted further investigation, in which we addressed the following questions: (1) What trends may be discerned in the test ordering behaviour of general practitioners over time at the Maastricht diagnostic centre? (2) Are the request patterns at the centre different from those of laboratories where no feedback is provided? (3) Do the changes observed in test ordering behaviour reflect advice given in the feedback? (4) What are the financial implications of any differences in request patterns resulting from the feedback?

\section{Subjects and methods}

Since 1985 general practitioners served by the diagnostic centre in Maastricht have been provided with feedback as critical biannual reports. These reports, which are based on analyses of request forms submitted in one month, discuss both quantitative and qualitative aspects of diagnostic test ordering. As the request forms offer clinical data on the patient (history, physical findings, suspected diagnosis, etc) it is feasible to assess the rationality of tests ordered.

The following data were gathered. Firstly, we surveyed the requests during 1983 to 1990 , then made an inventory of tests which were discussed in the feedback reports from 1985 to 1990 . We also obtained data on test requests during 1983 to 1989 in another Dutch region where a laboratory processed requests for diagnostic tests from general practitioners. That reference laboratory (referred to here as laboratory A) and its region were comparable to the Maastricht diagnostic centre and its catchment area with regard to the degree of urbanisation and the average family practice size. The Maastricht diagnostic centre and laboratory A processed virtually all requests for tests from general practitioners in the two regions. General practitioners in the region served by laboratory A received no feedback of the kind described in this paper.

Of all tests that were available, there were 46 that could be requested from both the diagnostic centre in Maastricht and laboratory A. The two centres were compared for these 46 tests, which are listed in the table. Eleven tests were discussed in virtually all reports to general practitioners, often more than once (see table). Hence any influence of feedback on test ordering behaviour should have been particularly evident for these tests.

The data were analysed with respect to the total number of requests for the 46 tests available in both centres, the number of requests for tests discussed frequently in feedback reports (see table) and the number for tests that were not, and several typical examples from the group of tests discussed frequently. To assess the economic implications we also carried out a financial appraisal, in which costs with and without feedback were compared.

To ensure anonymity of laboratory A all the data presented were indexed to the level of the Maastricht diagnostic centre in 1984. Beginning with 1985 we used the $\chi^{2}$ test to examine the data for each year to see whether differences were significant. ${ }^{18}$ The request figures for each year were compared with those for 1984. Figures for 1984 counted as zero measurement.
Correspondence to:

Dr Winkens.

BMF 1992;304: 1093-6 


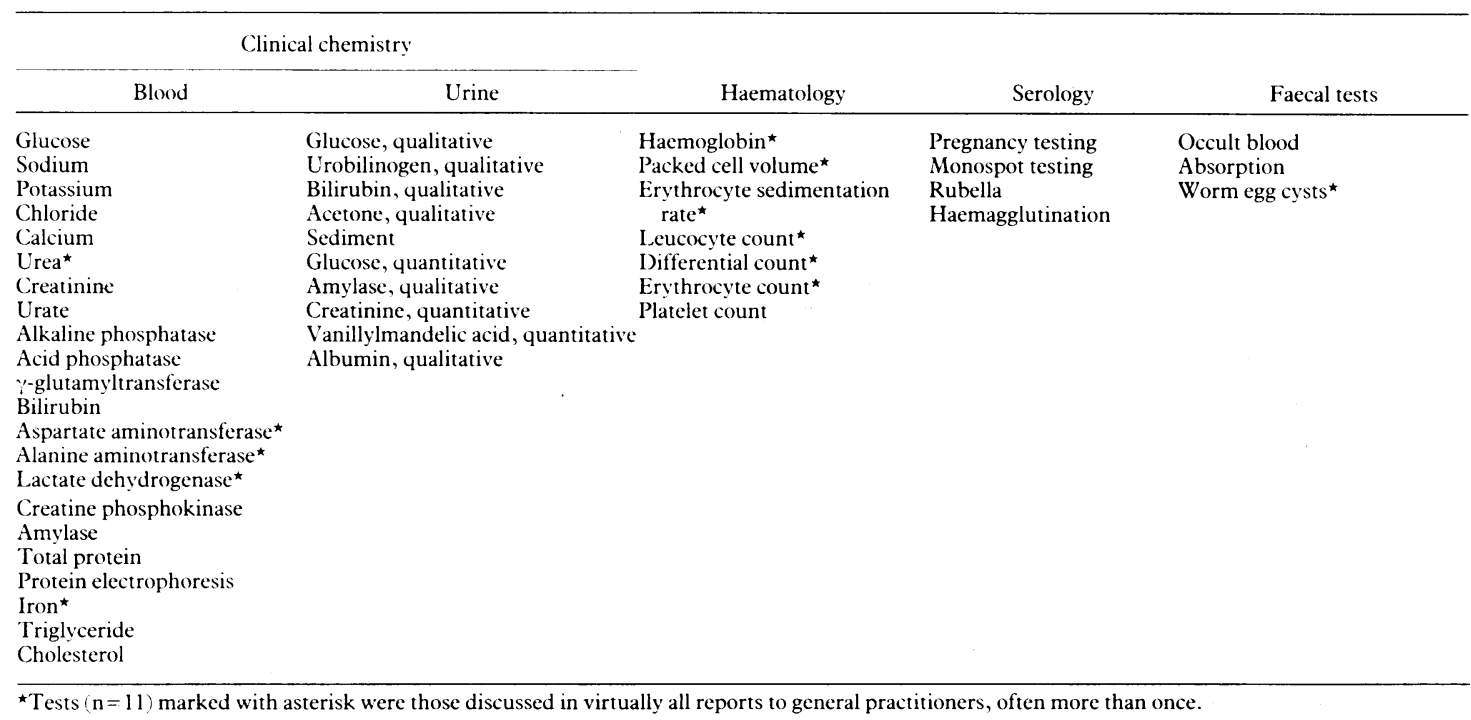

\section{Results}

Figure 1 illustrates the request trends at the Maastricht diagnostic centre with respect to both the total number of requests yearly and requests for the 46 tests also available in laboratory A. Figure 2 shows the yearly numbers of requests for the 46 tests alone at the two centres and in laboratory A with the data indexed to the number of patients in the Maastricht diagnostic centre's region (curve $x$ ).

Though the numbers of requests at the two centres were commensurate with the size of the patient population, the number of requests in the Maastricht diagnostic centre was considerably lower even before feedback was started (fig 2). The reduction in number of requests for the 46 tests at the Maastricht diagnostic

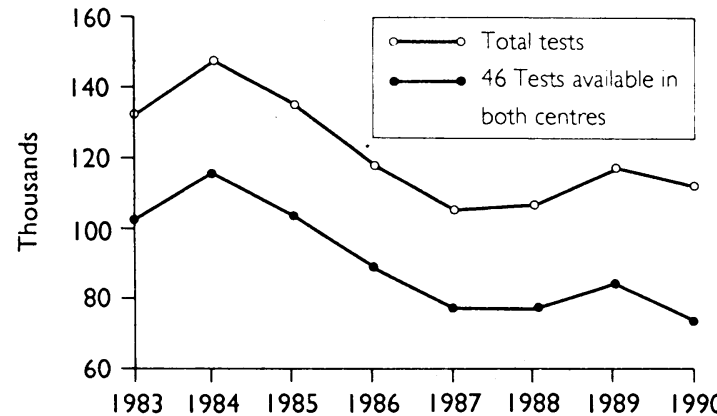

FIG 1 - Total numbers of tests requested yearly at Maastricht diagnostic centre and numbers of requests for 46 tests also available in laboratory $A$

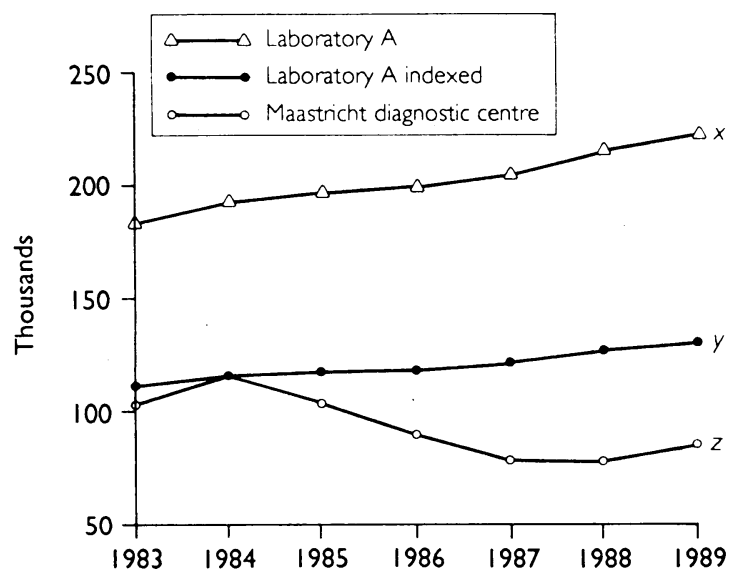

FIG 2 - Yearly numbers of requests for 46 tests available in Maastricht diagnostic centre and laboratory $A$ and in laboratory $A$ with data indexed to numbers of patients in Maastricht diagnostic centre region (curves $x, y, z$; see text)
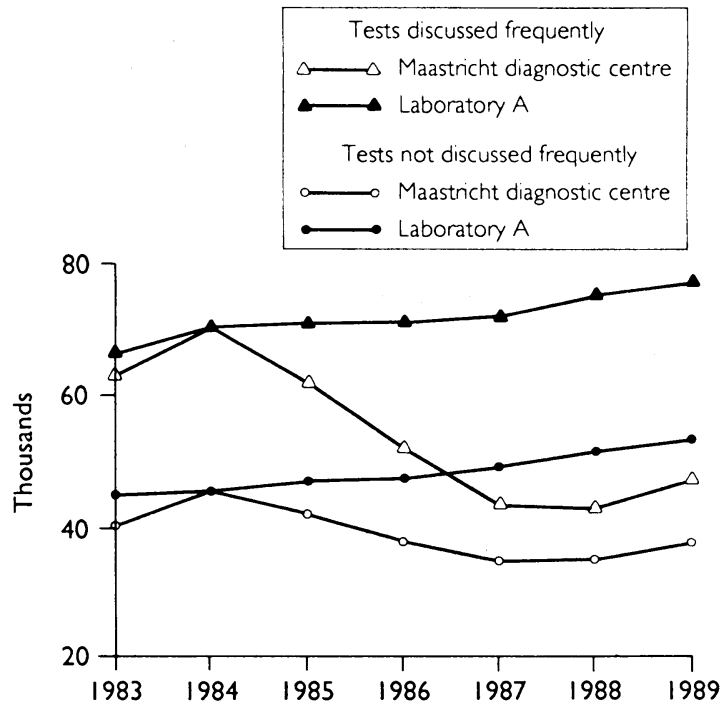

FIG 3 -Forty six tests available in two centres classified by whether they were $(n=11)$ or were not $(n=35)$ discussed in feedback

centre was clearly noticeable from 1985 onwards (curve $z$ ). By contrast, the number of tests ordered from laboratory A increased continually. The differences were significant $(p<<0 \cdot 001)$. Even when numbers for the two laboratories were standardised for 1984 (curve $y$ versus curve $z$ ) differences remained significant $(\mathrm{p}<<0.001)$.

Figure 3 shows the 46 tests available in the two centres classified by whether they were $(n=11)$ or were not $(n=35)$ frequently discussed in the feedback. Requests for the 11 tests frequently discussed are thus compared for the two laboratories. The effect of feedback was striking for these tests (for every year $\mathrm{p}<<0.001$ ), but requests for the 35 tests not discussed frequently also decreased significantly in Maastrich $(\mathrm{p}<0.001)$.

On examining data for Maastricht alone with respect to request trends for tests frequently discussed in the feedback and tests that were not dealt with in the reports, the decrease was seen to be most prominent for tests that were discussed (fig $3 ; \mathrm{p}<<0 \cdot 001$ ).

To illustrate the effect more clearly we elaborated a few examples that we expected would reveal a large impact of feedback (fig 4). These were tests stated repeatedly to be inappropriate under the given circumstances-namely, urea, lactate dehydrogenase, and haemoglobin measurements. These were compared with creatinine measurement, a test not designated 

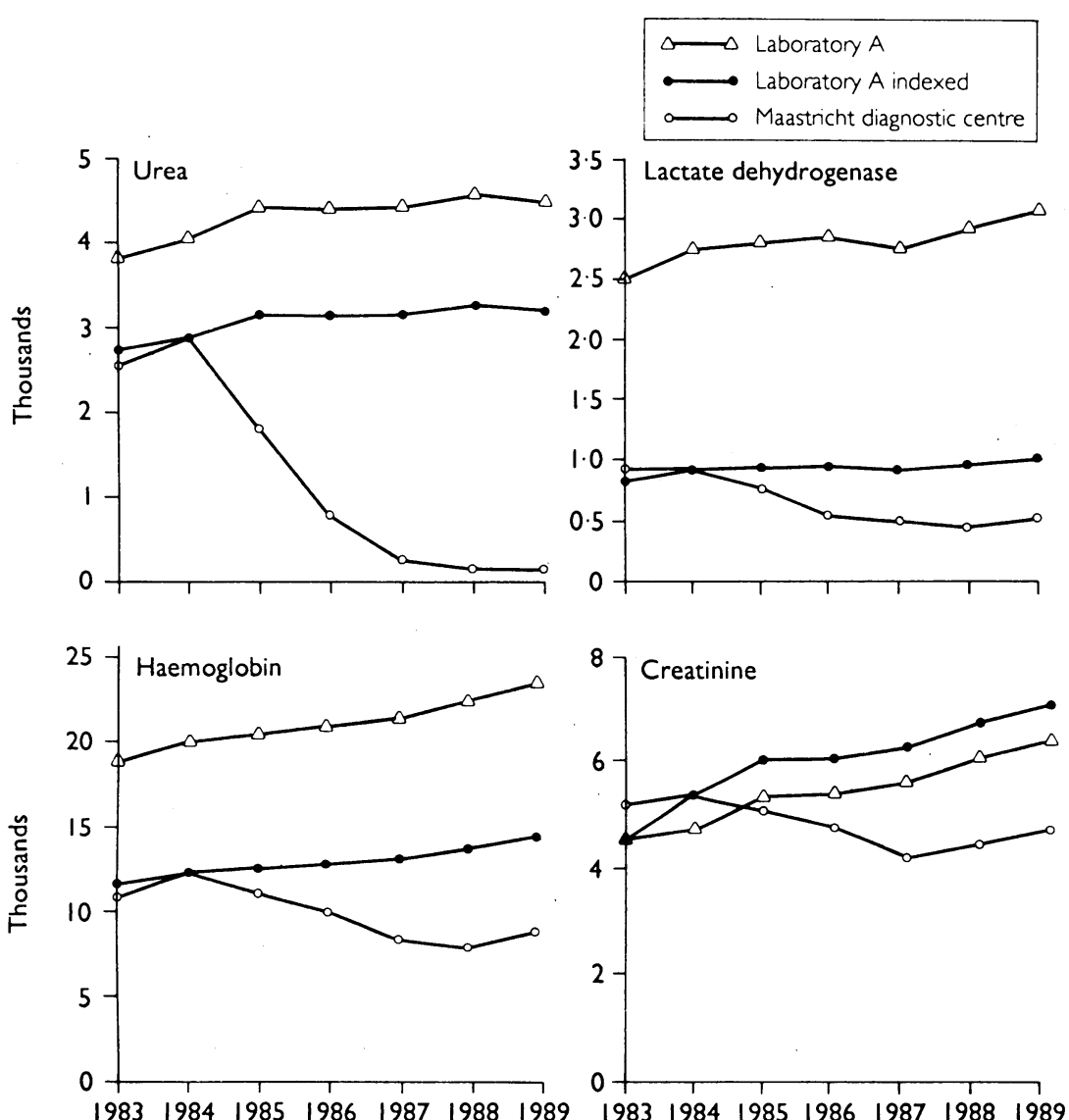

FIG 4-Four examples of influence of feedback in Maastricht diagnostic centre and laboratory $A$ as inappropriate. The differences between the two laboratories were unequivocal $(\mathrm{p}<<0 \cdot 001)$.

The observed changes had major financial implications. For all diagnostic procedures (endoscopy not included) expenditure of the Maastricht diagnostic centre increased from about $\mathrm{fl} 1.59 \mathrm{~m}(£ 500000)$ in 1980 to $\mathrm{fl} 2 \cdot 14 \mathrm{~m}(£ 673000)$ in 1984 . Nationally, the annual increase was $8 \%$. On the basis of this rate of increase the expenditure would have amounted to fl $3.39 \mathrm{~m}(£ 1.07 \mathrm{~m})$ in 1990 . In fact, costs were fl $2.13 \mathrm{~m}$ $(£ 670000)$. Included were the extra costs of the data processing and analyses for the feedback reports (fig 5).

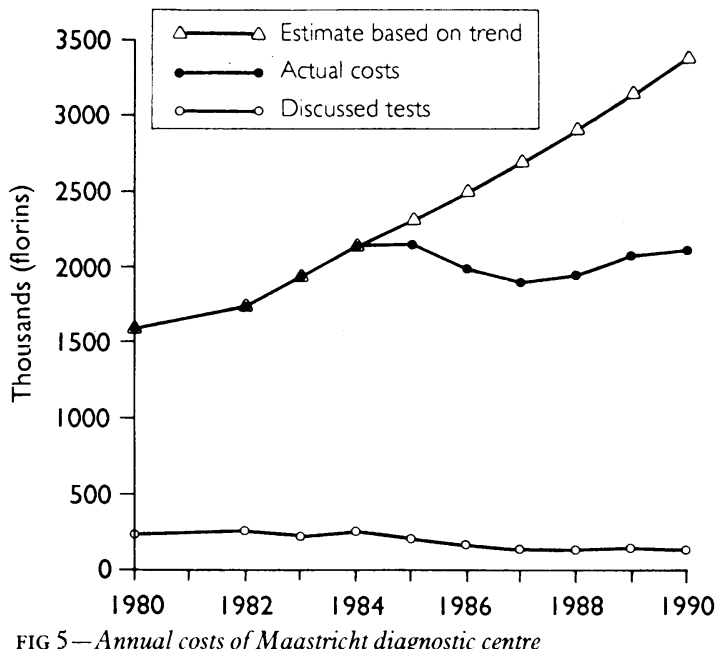

\section{Discussion}

The number of tests ordered from the diagnostic centre in Maastricht decreased sharply after 1984. Comparison with laboratory A indicated that this reduction was specific to the Maastricht centre and was especially evident for those tests that were discussed frequently in the feedback reports.
A potential effect of feedback was assessed mainly by retrospective comparison of the Maastricht diagnostic centre with laboratory A. Distortion may have occurred as a result of selection bias-that is, the 46 tests studied as available in both centres may have offered too favourable a picture of the Maastricht diagnostic centre. Figure 1 shows, however, that they were a representative sample.

Figure 3 illustrates that the number of requests for tests that were not discussed also decreased in Maastricht. This could be explained by a general learning effect arising from the feedback. Conversely, the number of requests in 1988 and 1989 increased slightly. The test ordering behaviour seemed to reach a minimal level in 1987. Probably an increase in the number of requests might have been expected, given the reinforcement of primary health care in recent years - for instance, with regard to the management of non-insulin dependent diabetes mellitus by general practitioners. In addition, some tests-for example, cholesterol estimations-have become increasingly popular among the general population. In 1990 the number of requests decreased again. We had no data from laboratory A for 1990, and therefore these figures could not be compared in our study.

The 11 tests discussed in the feedback were requested much more often than the 35 tests not discussed. The reason is that the 11 tests comprised those which individually have by far the highest request numbers.

In 1983 and 1984 roughly $40 \%$ fewer tests were ordered in the Maastricht region than in the region of laboratory A. A possible explanation may be that soon after the Maastricht diagnostic centre was set up a request form was designed that listed only a relevant selection of tests-and we know that the layout of request forms influences test ordering behaviour. ${ }^{19}$ Another explanation may be that compared with the Maastricht diagnostic centre laboratory A provided better services and was more accessible to general practitioners and patients. However, we could not deduce any differences of that kind from the data that were available. It seems unlikely that any other confounders besides the feedback influenced the test ordering behaviour.

Theoretically, the saving in costs may have been annulled by an increase in the number of referrals. Overall referral patterns did not indicate that this had occurred. Finally, although p values indicated highly significant differences, this was partly due to the large number of requests.

This study was granted by the Dutch Minisiry of Public Health. We thank the members of its guiding committee for their assistance during the study.

1 Fraser CG, Woodford FP. Strategies to modify the test-requesting patterns of clinicians. Ann Clin Biochem 1987;24:223-31.

2 Horder J, Bosanquet N, Stocking B. Ways of influencing the behaviour of general practitioners. I R Coll Gen Pract 1986;36:518-21.

3 Grossman RM. A review of physician cost-containment strategies for laboratory testing. Med Care 1983;21:783-801.

+ Eisenberg JM, Williams SV. Cost containment and changing physicians' practice behavior. FAMA 1981;246:2195-202.

5 Sprij B, Casparie AF, Grol R. Interventiemethoden om een verandering in de medische praktiikvoering te bewerkstelligen; wat is effectief? Ned Tijdschr Geneeskd 1989:133:1115-8.

6 Ende J. Feedback in clinical medical education. JAMA 1983;250:770-81.

7 Berwick DM, Coltin KL. Feedback reduces test use in a health maintenance organization. FAMA 1986;255:1450-4.

8 Tierney WM, Miller ME, McDonald CJ. The effect on test ordering of informing physicians of the charges for outpatient diagnostic tests.

9 McDonald CJ, Hui SL, Smith DM, Tierney WM, Cohen SJ, Weinberger M, et al. Reminders to phssicians from an introspective computer medical record. A two-year randomized trial. Ann Intern Med 1984;100:130-8.

10 Davidoff F, Goodspeed R, Clive J. Changing test ordering behavior. A randomized controlled trial comparing probabilistic reasoning with costcontainment education. Med Care 1989:27:45-58

11 Rhyne RL, Gehlbach SH. Effects of an educational feedback strategy on physicians' utilization of thyroid function panels. F Fam Pract 1989;8: $1003-7$

12 Mugford M, Bantield P, O'Hanlon M. Effects of feedback of information on clinical practice: a review. BM7 1991;303:398-402. 
13 Eisenberg JM. An educational program to modify laboratory use by house taff. F.Med Educ 1977;52:578-81.

14 Fowkes FGR, Evans KT, Hartley G, Nolan DJ, Roberts CJ, Davies ER, et al Multicentre trial of four strategies to reduce use of a radiological test. Lancel $1986 ; 1: 367-70$

15 Marton KI, Tul V, Sox HC. Modifying test-ordering behavior in the outpatient medical clinic. A controlled trial of two educational interventions. Arch Intern Med 1985;145:816-21.

16 Bareford D, Hayling A. Inappropriate use of laboratory services: long term combined approach to modify request patterns. BMF 1990;301:1305-7.
17 Pop P, Winkens RAG. A diagnostic centre for general practitioners: results of individual feedback on diagnostic actions. $\mathcal{F} R$ Coll Gen Pract 1989;39: 507-8.

18 Fleiss JL. Statistical methods for rates and proportions. 2nd ed. New York: John Wiley and Sons, 1981.

19 Zaat JOM, van Eyck JThM, Bonte HA. Mag het ook een testje minder? De invloed van verandering van het aanvraagformulier voor laboratorium onderzock. Huisarts en Wetenschap 1991;34:72-7.

\title{
Study of diagnostic accord between general practitioners and an ophthalmologist
}

\author{
J H Sheldrick, S A Vernon, A Wilson
}

\section{Abstract}

Objectives-To identify diagnostic accord and disagreement between general practitioners and an ophthalmologist and thereby determine how undergraduate and non-specialist postgraduate ophthalmic training could be improved.

Design-Comparison of diagnosis of presenting conditions by general practitioners and one ophthalmologist in patients consulting general practitioners for ophthalmic problems during March 1989 to February 1990.

Setting - 12 general practices in west Nottingham

Patients-1474 patients presenting to the study general practitioners with new ophthalmic conditions or new episodes of recurrent conditions.

Main outcome measures-Diagnoses of general practitioners and ophthalmologist.

Results $-1121(76 \%)$ of patients with eye problems agreed to see the ophthalmologist and most were seen within three days. Sufficient data for comparison were available on 1103 patients. Diagnostic agreement was found in 638 cases $(58 \%)$, but potentially serious misdiagnosis was found in only 15 cases; management in three of these cases would have ensured later identification. Most commonly confused conditions were infective and allergic conjunctivitis, blepharitis, and dry eyes. General practitioners assessed visual acuity in only 114 cases yet eight of the 15 patients seriously misdiagnosed had reduced acuity, an important diagnostic sign.

Conclusions-Most ophthalmic disease seen in general practice does not require specialised equipment for diagnosis. Most cases of misdiagnosis have no serious consequences for the patient. Undergraduate and postgraduate training in ophthalmology should ensure that common conditions can be easily differentiated and more serious conditions identified and referred.

\section{Introduction}

Eye disease is responsible for over $2 \%$ of general

Department of General Practice and Academic Unit of Ophthalmology, University of Nottingham, Nottingham

J H Sheldrick, research fellow

S A Vernon, senior lecturer

A Wilson, lecturer

Correspondence to:

Mr J H Sheldrick,

Department of

Ophthalmology, Leicester

University, Leicester Royal

Infirmary, Leicester.

BMF 1992;304: 1096-8 on general practitioners.

How accurately do these hospital based studies reflect general practitioners' ophthalmic abilities? General practitioners manage most ophthalmic prob- lems presenting to them. Referred cases are likely to represent those in which the practitioner has uncertainties or where ophthalmic management is thought necessary. In a study of eye disease presenting to a London community health centre ${ }^{2} 30$ patients were examined by an ophthalmologist as well as their general practitioner. Agreement was found in only 12 cases.

We conducted a study to assess the ophthalmic diagnostic accuracy of general practitioners in a larger sample to determine any important disagreements and thereby provide useful information for the planning of undergraduate and postgraduate ophthalmic training. The study is not an attempt to show that an ophthalmologist using specialised equipment can identify more eye conditions than general practitioners.

\section{Subjects and methods}

Between March 1989 and February 1990 we studied patients of 17 general practitioners in the western sector of the city of Nottingham to examine the rates of ophthalmic disease in a defined population of 36018 .

Patients presenting to their general practitioner with eye problems were recorded by practitioners in books of questionnaires. Practitioners were asked to record their working diagnosis, investigations, treatment, and follow up arrangements. Specific questions included whether visual acuity was assessed or fluorescein staining used. Patients diagnosed by the general practitioner as having either a first episode of an ophthalmic condition or a new episode of a recurrent ophthalmic condition were asked by the practitioner if they would see an ophthalmologist as part of a research project. Patients who agreed were seen by a single ophthalmologist (JHS) as soon as possible after presentation to the general practitioner at the general practitioner's surgery or by domiciliary visit. The ophthalmologist carried out a problem oriented ophthalmic examination using a $3 \mathrm{~m}$ Snellen chart, Perkins hand-held tonometer, portable slit lamp, and diagnostic pharmaceuticals as required. Laboratory investigations were not used, and all diagnoses were based on history and clinical examination as in previous epidemiological studies of eye disease presenting to emergency departments. ${ }^{10}$ None of the general practitioners had worked in ophthalmology after graduation.

Questionnaires completed by general practitioners and ophthalmologist on each patient at the time of consultation were analysed with the statistical package SPSSX. The general practitioners' and ophthalmologist's diagnoses of the presenting condition were then compared. Diagnoses were considered to agree if the presenting condition diagnosed by the general practitioner was confirmed by the ophthalmologist. When conjunctivitis was the only diagnosis specified by the general practitioner the type of conjunctivitis implied 